\title{
Barrage sur la ligne de fuite. Considérations sur Nietzsche et la prudence philosophique
}

\author{
DALIE GiROUX
}

«Le Diable n'est que le loisir de Dieu au septième jour »

(Nietzsche, Ecce Homo).

Ce texte présente une tentative de trouver une notion de «prudence philosophique » chez Nietzsche. Il procède de la critique d'une contribution de Daniel Tanguay dans laquelle l'auteur s'oppose au diagnostic posé par certains exégètes de l'œuvre de Leo Strauss d'une certaine proximité entre la pensée du maître américain et celle du prophète allemand. Ainsi, dans la contribution de Tanguay, une interprétation nietzschéenne de Strauss par des straussiens (en l'occurrence, celle de Laurence Lampert) est renvoyée dos-à-dos à une interprétation straussienne de Nietzsche (celle de Tanguay). L'argument principal, visant à montrer la distance entre Strauss et Nietzsche, tient en cette idée que la prudence, qui est le propre de la sagesse philosophique et qui est au cœur de la pensée de Strauss, est absente chez Nietzsche. Leo Strauss, puisque philosophiquement prudent, ne peut être associé à un penseur (Nietzsche) qui au terme s'est révélé, selon les mots de Tanguay, dogmatique. La démonstration proposée est soustendue par une thèse sur la figure du philosophe et sur la nature de la vie philosophique. Celle-ci, lorsque pesée à l'aune de l'œuvre nietzschéenne, offre l'occasion d'aborder des questions qui participent du travail de signification du texte nietzschéen. 
Développant une argumentation contre la conclusion de Daniel Tanguay, je veux préciser les conditions de possibilité et le sens d'une prudence philosophique nietzschéenne. En particulier, la recherche d'une notion de prudence chez Nietzsche demande d'explorer d'une part une possible distinction entre eros et volonté de puissance, et d'autre part une possible distinction entre nature et volonté de puissance. Je souhaite aborder ces thèmes à partir du problème posé par le texte de Tanguay, dans l'espoir d'enrichir cette aire de débat qui concerne au fond la possibilité et la valeur de la philosophie. Outre ma propre lecture du texte nietzschéen, j'utiliserai l'interprétation de Daniel Conway, qui aborde la question de la possibilité de la philosophie à l'ère du nihilisme dans le contexte contemporain, ainsi que, de manière plus diffuse, la lecture de Gilles Deleuze, que j'estime être l'interprète de Nietzsche le plus pénétrant et le plus stimulant.

\section{La vie philosophique}

Il s’agit pour Daniel Tanguay, dans sa lecture de certains interprètes de Leo Strauss, de défendre ce dernier de l'accusation de nietzschéisme. Une des clefs de l'argumentation de l'auteur consiste à montrer, en se servant des arguments anti-nietzschéens de Strauss lui-même, que Nietzsche, contre sa propre intention, a développé une métaphysique. C'est là, est-il entendu, une contradiction et une imprudence.

La volonté de puissance et l'Éternel retour, écrit Tanguay, ont la prétention de décrire les déterminations les plus essentielles de l'Être ou, pour utiliser le langage straussien, du Tout. Cette prétention est purement dogmatique pour Strauss, puisque l'homme en tant que partie du Tout ne peut saisir la totalité du Tout (126). 
Le moteur de la vie philosophique serait révolution chez Nietzsche et idéalisme prudent chez Strauss.

Cet argument, archè du texte de Tanguay, est notamment utilisé en contrepoint à l'argument de Lampert selon lequel Nietzsche aurait une conception straussienne (ou platonicienne ce qui, nous disent Lampert et Tanguay, revient au même) de la vie philosophique. Tanguay accorde à Lampert une partie de son raisonnement :

Le « retour à la nature » proposé par Nietzsche est un retour à une nature qui tend à son accomplissement total en la personne du philosophe. Lampert a raison de dire que sur ce point «la vision nietzschéenne du philosophe n'est pas différente de celle de Platon. »... Strauss est donc nietzschéen dans la mesure où il cherche comme Nietzsche à reconquérir les conditions naturelles qui donnent naissance au philosophe platonicien (119).

L'homme complémentaire nietzschéen, Tanguay s'accorde également avec Lampert là-dessus, aurait pour tâche «une défense de la vie philosophique : elle est le mode de vie le plus noble et elle justifie le reste de l'existence » (118).

C'est plutôt à la deuxième partie de l'argument de Lampert que s'en prend Tanguay, argument selon lequel Strauss, dans sa stratégie exotérique, n’a pas assumé la nécessité du rétablissement de l'ordre naturel hiérarchique au sommet duquel, en l'absence de Dieu, se trouve le philosophe. Pour Tanguay, cette interprétation impute à Strauss un athéisme qu'il n'aurait jamais assumé et qui relèverait d'un dogmatisme qui minerait la philosophie bien plus que ne le ferait la suspension d'un jugement final sur la question de la Révélation :

la véritable philosophie, la philosophie zététique, doit suspendre son jugement sur cette question [le rejet d'un Dieu biblique], tout en prenant conscience que cette suspension du jugement affaiblit grandement sa prétention à se présenter comme la meilleure vie (121).

Devant cet argument, je veux suggérer dans un premier temps que Lampert, Strauss et Tanguay interprètent incorrectement la figure du «philosophe » nietzschéen. Je m’en tiendrai ici 
dans un premier temps à quatre illustrations qui formeront le contexte dans lequel j'aborderai les autres questions soulevées par la contribution de Daniel Tanguay. Ces vignettes suggèrent que Nietzsche n'est ni athée, ni apodictique, ni propagandiste de la vie philosophique, ni peut-être même philosophe, à tout le moins au sens platonicien.

Premièrement, Nietzsche, s'il est un dogmatique, et je reviendrai sur cette question, n'est clairement pas un athée. J'aimerais à ce sujet attirer l'attention sur le $\S 24$ de la troisième dissertation de la Généalogie de la morale. Nietzsche y commente l'athéisme, d'une manière que Strauss-Tanguay ne lui disputeraient pas, puisque l'auteur allemand y utilise précisément cet argument du dogmatisme de l'athéisme pour le réfuter comme dernière philosophie idéaliste :

Ils se croient tels en effet, ces «incroyants » (cela ils le sont tous); on dirait que c'est précisément en quoi consiste tout ce qui leur reste de foi : être les adversaires de cet idéal, tant ils sont graves et passionnés à ce sujet dans leurs paroles et dans leurs gestes, - serait-ce une raison pour que ce qu'ils croient soit vrai?

Faut-il croire ce qu'un auteur dit de sa propre pensée? Nietzsche se félicite de ne pas être celui qui devra « casser cette noix », en référant à sa propre pensée... Le $§ 24$ indique à tout le moins que Nietzsche était au fait de cette difficulté de la pensée par-delà bien et mal, et qu'il est légitime d'examiner très attentivement les preuves de l'accusation de dogmatisme portée contre Nietzsche par Strauss-Tanguay. Ce qu'il me semble, en guise d'entrée en matière, c'est que la vérité n'est pas une divinité chez Nietzsche, ce qui ne signifie pas que celle-ci (la vérité) ou celle-là (la divinité) n'ont pas de valeur. Il y a clairement des vérités chez Nietzsche, lui qui écrit dans Ecce Homo (IV, § 1): «la vérité parle par ma bouche ». II y a aussi des divinités : Dionysos, Zarathoustra, Chaos, Éternité. Celles-ci fonctionnent, propose Nietzsche, à la manière du ciel grec, comme des représentations des affects qui permettent de moduler la volonté de puissance de la manière la plus satisfaisante pour la vie elle-même. Les divinités mettent en 
scène l'incarnation des puissances de la vie et de la mort, horizon de toute expérience : le viol et l'amour, le carnage et la joute. Je me permets de citer longuement Nietzsche sur la fonction des divinités.

Ils prenaient, écrit Nietzsche à propos des Grecs, ces côtés trop humains comme quelque chose d'inévitable et, au lieu de les avilir, préféraient leur conférer une sorte de droits de second ordre en les insérant à leur place dans les coutumes de la société et du culte; bien mieux, ils nommaient divin tout ce qui a quelque puissance dans l'homme, ils l'inscrivaient sur les murs de leur ciel. Ils ne renient pas l'instinct naturel qui s'exprime dans les mauvaises qualités, mais le mettent à sa place, dans les limites de certains cultes, de certaines journées, ayant inventé assez de précautions pour pouvoir donner un cours aussi inoffensif que possible à ces flots torrentueux. C'est là toute l'indépendance d'esprit de l'Antiquité en matière de morale. Au mal, au scabreux, aux traits de bestialité arriérée, à ce barbare préhellénistique et asiatique qui continuait à vivre au fond de la nature grecque, on accordait une détente modérée et l'on n'aspirait pas à son anéantissement complet (Humain trop humain II, «Opinions et sentences mêlées », § 220).

Il n'y va pas, dans cette version de la relation entre Dieu et Nietzsche, autant de l'athéisme que d'une sagesse de laisser coexister les inéluctables (et non principiels!) contraires, tel que cela est suggéré dans cette apologie du polythéisme, et tel que cela est recréé dans le style de l'œuvre de Nietzsche. Ailleurs, Nietzsche suggérera qu'il ne s'agit pas de dire que Dieu est une erreur en soi, mais plutôt qu'il y a des manières plus nobles de servir cette idée — on pourrait dire déjà cette prudence (voir Généalogie II, § 23).

Deuxièmement, Nietzsche exerce face au langage, et le sien en particulier, une prudence exemplaire. Son travail est parsemé d'avertissements au lecteur, selon lesquels l'écriture est éminemment problématique, le système une duperie. À propos de Zarathoustra : «peut-être vous a-t-il dupé ... ». Il y a dans l'écriture nietzschéenne un perpétuel renversement de l'Idole que peut constituer l'écriture, en particulier la sienne. Il est révélateur que Nietzsche, ayant parfois honte de sa qualité d'écrivain, estimait que son autorité allait un jour tenir au fait qu'on ne le comprendrait pas. Le corollaire de cette affirmation est que si on le comprenait, il n'y aurait pas 
d'autorité qui tienne, non pas à tout le moins une autorité qui soit pétrifiée par les mots. Il faut savoir se garder, dit un passage particulièrement perfectionniste du Gai savoir. Le statut de sa prétendue « philosophie », au sein même de cette philosophie, est fragile, fragilisé. Être un satyre plutôt qu'un saint. Zarathoustra renvoie ses disciples, Nietzsche répétant ce passage d'Ainsi parlait Zarathoustra dans la préface d'Ecce Homo. Il me semble que par ce geste littéraire de 1885 répété en 1888, Nietzsche évite, s’il ne se prive pas de poser sa propre vie en modèle, de se poser en «sobre héros de la révolution», une révolution philosophique par laquelle la hiérarchie naturelle qui se cache sous le vocable de la «noblesse » serait remise en selle dans la pensée de Nietzsche. Nietzsche me semble être son propre saboteur — et joyeux.

Troisièmement, il n'est pas certain que « la vie philosophique soit au-dessus de tout», avec l'être complémentaire. Il me semble que c'est le dépassement dont les êtres exemplaires sont capables (qui sont philosophes, mais aussi artistes, saints, tyrans) qui est cher à la vision nietzschéenne, et non pas ces hommes pour eux-mêmes qui, mêmes exemplaires, ne sauraient qu'être un pont. Ces hommes complémentaires, quête érotique de la volonté forte, se dépassent eux-mêmes. Ils se réinventent. Dans ce dépassement, ce don, ils créent comme par accident la culture, produisent la communauté qui se reconnaît, quête érotique de la volonté faible, dans ce don. Cette culture est le siège de la création d'êtres exemplaires. C'est du moins ainsi que l'interprète Daniel Conway:

the philosopher creates a community only indirectly and unwittingly, through his expenditure of the excess affect required to turn the hammer on himself. He thus becomes a sign unto himself, irrepressibly projecting his self-directed legislations into the public space that surrounds him (293-294).

N'est-ce pas la dialectique morale, le mouvement symbolique, qui importe à Nietzsche bien plus que la philosophie et la vie du philosophe? N'est-ce pas le mouvement pour le mouvement qui 
est valorisé (au nom de l'espoir, une folie, et non pas au nom de la nature), plutôt que la vie par laquelle se contemplent les formes pures? Quand il y a trop de raison, la folie est une raison supérieure, disait Nietzsche.

Le livre premier du Gai savoir, et particulièrement les cinq premières sections, me semble contenir plusieurs indications précieuses à cet effet. L'aphorisme 4 insiste notamment sur la valeur du «mal », c'est-à-dire de la contradiction morale culturelle, comme source d'innovation. L'humanité n'est que l'expression d'une création, la nature humaine (§ 1). La noblesse (§ 3) est la quête passionnelle de cette innovation, et l'idée de «vérité absolue » (§ 5), particulièrement dans la pensée politique, nuit à l'émancipation, qui semble définie comme le fait même de la capacité de l'humanité d'innover en matière morale (culturelle). L'humain est ici un «être-pour». La vie n'est pas vie-pour-le-philosophe, c'est le philosophe qui est philosophepour-la-vie. Les conditions de génération des conditions culturelles d'être-pour-la-vie, d'hommes exemplaires, sont l'horizon de toutes les noblesses - et même pas encore leur fin. C'est l'éternité qui est éternelle (mystère du monde), non pas la philosophie, non pas le philosophe.

Quatrièmement, il me semble que le philosophe n'est plus tellement philosophe, chez Nietzsche. Comme l'écrit Conway :

In praising philosophers, however, he does not have in mind those narrow specialists, bookish scholars and petty «laborers» who are popularly identified as «philosophers ». Genuine philosophers are those intrepid thinkers who legislate a vision of human perfectibility, a goal toward the attainment of which resources of humankind might be profitably gathered and directed (288).

Il ne s'agit pas tellement pour le philosophe de «connaître », chez Nietzsche, comme de « chasser le pouvoir », tel un mage et un guerrier. La contemplation (tout comme l'objectivité et la recherche de la chose en soi) n'est pas une vertu chez Nietzsche, elle maladie ou mensonge. Ainsi, s'il y a un philosophe chez Nietzsche, ce n'est pas un philosophe platonicien. Il n'y a pas 
de connaissance pour la connaissance puisqu'il n'y a pas de formes pures autres qu'effets de l'existence, il n'y a de connaissance qu'au service de la vie, une position sur le savoir que Nietzsche a conservée et cultivée dans toute son œuvre écrite. Il s'agit peut-être plutôt de conaître. Gilles Deleuze a cette formule à propos de ce qu'est penser qui répond le mieux à mon avis à l'injonction nietzschéenne : penser est ce qui se passe à la jonction du voir et du parler. «Errance dans l'interdit » est la pratique philosophique chez Nietzsche, elle est expérience du corps (pas de café le matin, un usage modéré des livres, ne faire confiance qu'aux pensées qui viennent en marchant), don total et égoïsme pur, passion, confiance : «ce qui est bon pour moi est bon en soi ».

Les images guerrières sont multiples dans le texte nietzschéen publié. Elles ont fait l'objet d'interprétations militaristes, en général superficielles. Le philosophe, «Prince hors-laloi », est un guerrier, un souverain, un législateur. Il maîtrise le secret de la culture — il a eu le courage de poser son regard au fond de l'abîme. Chasser le pouvoir, c'est développer la capacité de donner de la valeur. Le pouvoir de donner de la valeur est un don, qui n'est pas un talent comme une capacité développée par le fruit tardif du processus de civilisation (l'individu souverain), et qui se présente comme la récusation même de l'échange. Le don court-circuite le fait même de la fabrication anthropologique de la conscience, de la promesse et au terme, de l'individu souverain : il abolit l'échange, forme primitive du rapport humain (le marché et la justice). C'est par le don que l'individu est souverain. ${ }^{1}$

Cette capacité est développée par les «philosophes du futur », figues mûres sur lesquelles la brise nietzschéenne souffle, qui ne seront pas exécutants de la Nature, mais fine fleur du processus de civilisation. Manipulateurs de l'Invisible, de l'ombre du Visible, ils s'approprient les forces de la domestication et peuvent donner — à travers la promesse à soi-même et par la 
production d'un excédent érotique - sa forme au plastique culturel. Ces créateurs, ces êtres délivrés des rapports de force originels et inconscients, ces individus capables de projets, ces voyageurs et leurs ombres, seront avant tout des expérimentateurs de leur propre dualité, de leur propre finitude, de leur propre dépassement (père et mère, mort et vie, ombre et lumière, mal et bien en éternel retour). Par accident érotique, comme le veut ce mythe selon lequel le monde est né de l'éjaculation d'un dieu se masturbant, les créateurs donneront naissance à la culture. ${ }^{2}$ Il me semble, et c'est une hypothèse que j'ai développée ailleurs (Giroux, "L'unité »), que le «philosophe du futur » est avant tout dans la pensée de Nietzsche le détenteur d'un secret antique, celui de la danse entre la vie et la mort, et celui de la parole comme performance civilisationnelle : « au début était le verbe».

\section{Le statut de la volonté de puissance chez le Nietzsche de maturité}

Une question demeure en suspens dans cette amorce d'une discussion sur la conception de la vie philosophique chez Nietzsche. Il s'agit d'établir si, effectivement, la pensée de Nietzsche est une métaphysique. Deux sous-questions relèvent de cette problématique, qui ont été élégamment dégorgées par Daniel Tanguay. La première porte sur la relation entre l'eros philosophique et la volonté de puissance, la seconde, sur la relation entre la nature et la volonté de puissance. Il faut répondre à ces deux sous-questions pour accomplir la tâche d'examiner le problème d'une éventuelle métaphysique nietzschéenne. Je veux mettre en perspective ces deux sous-questions en replaçant la discussion sur Nietzsche dans le vocabulaire nietzschéen. 


\section{1. Eros $e t$ amor fati}

La première question posée par Tanguay est la suivante, et elle relève de la discussion menée plus haut. Est-ce que l'eros philosophique platonicien est différent de la volonté de puissance nietzschéenne? Pour Daniel Tanguay, il y a effectivement une différence et cette différence, qui est posée en termes de degrés de «prudence», exprimerait la distance entre les visions respectives de Strauss et de Nietzsche. L'eros philosophique est, écrit Tanguay,

ce mouvement qui porte l'âme vers la connaissance et la sagesse. Il est essentiellement mouvement de l'opinion vers la science. Strauss ne nous dit pas qu'il y a correspondance entre ce mouvement de l'âme et le mouvement propre au Tout (127).

L’ordre éternel dans la quête de la vérité est «supposé » sans être donné pour évident (127). On suggère ici que, chez Nietzsche, cet ordre éternel serait donné pour évident (dogmatisme associé à l'idée de la volonté de puissance), plutôt que supposé (prudence juxtaposée par décision à l'eros philosophique). ${ }^{3}$

Une précision me semble ici s'imposer. La volonté de puissance, à propos de laquelle Nietzsche a bien écrit, dans Par-delà bien et mal, qu'elle est sa «thèse », recoupe une réalité existentielle plus large que l'eros philosophique, ce dernier étant dans le langage nietzschéen une région de la volonté de puissance, une volonté de vérité. Chez Nietzsche, cette volonté de vérité comme toute volonté de puissance lorsqu'elle est noble, est amor fati. Ainsi, ce qui correspondrait éventuellement chez Nietzsche à l'eros philosophique platonicien serait une volonté de vérité placée sous l'égide de l'amor fati. Tanguay explique que l'amor fati est l'expression même de la volonté de puissance, et par là il a tort (voir sa note 4). L'amor fati est une volonté de puissance, mais aussi, ce qui est fondamental, une noble volonté puissance. Cet amour est le critère même de la noblesse. Il faut ainsi déplacer ou préciser le questionnement, et 
demander : est-ce que l'eros philosophique du philosophe platonicien est différent de la volonté de vérité placée sous l'égide de l'amor fati du philosophe nietzschéen?

Ma réponse à cette question serait oui, mais il ne s'agit pas de la différence entre une quête de connaissance humble quant à sa raison (à défaut d'être humble quant à sa finalité : « le bien en soi ») et une réalisation dogmatique et criarde de la hiérarchie naturelle. C'est ainsi que le problème est posé dans le texte de Tanguay. Ce dernier donne sa propre définition de l'amor fati: «la reconnaissance, voire la promotion, de la thèse de l'inégalité naturelle entre les hommes. L'inégalité naturelle, condition sine qua non de la grandeur humaine, doit être voulue » (119). Comparons cette définition, straussienne, avec celle qu'en donne Nietzsche dans Ecce

\section{Homo :}

Je ne connais pas d'autre manière d'avoir commerce avec les grandes tâches que le jeu : c'est, pour indiquer la grandeur, une condition essentielle. ... Ma formule pour désigner la grandeur dans l'homme, c'est l'amor fati : que personne ne veuille rien d'autre autrement, ni en avant, ni en arrière, ni dans les siècles des siècles. Ne pas seulement supporter la nécessité, encore moins se la dissimuler — tout idéalisme est une manière de mentir devant la nécessité —, mais l'aimer ... (I, § 10).

Et encore : «ce qui est inéluctable ne me blesse pas; l'amor fati est le fond de ma nature » (Ecce

Homo III, «Le cas Wagner », § 4). C'est cette «lucidité » qui est le critère de la grandeur chez Nietzsche, et c'est aussi ce qui est son hubris : «mon danger c'est le dégoût de l'homme » (Ecce Homo IV, § 6).

Je me contenterai ici de faire deux commentaires sur la similarité et la différence de l'eros philosophique et de l'amor fati, commentaires dans lesquels je souhaite comprendre la pensée de Nietzsche à la lumière des écrits de $1888 .{ }^{4}$ Je m’oppose par là à l'habitude de StraussTanguay de lire Nietzsche à l'aune du projet civilisationnel des années 1872-1877 qui, s'il est plus à même de permettre à l'histoire de la philosophie de formuler une «politique de 
Nietzsche », occulte d'une manière trop utile l'évolution de la pensée de Nietzsche. Cette dernière, j'y reviendrai, me semble passer d'une esthétique politique à une «éthique matérialiste » d'un genre inouï, encore que cela n'est peut-être pas encore un bon terme pour qualifier cette expérience nietzschéenne de la pensée.

Premièrement, il me semble que l'eros platonicien n'est pas exactement amor fati, parce que l'objet du désir de l'eros est «connaissance » et «sagesse », tel que l'écrit Daniel Tanguay, alors que l'objet du désir de l'amor fati est tout simplement «ce qui est », «ce qui arrive » à chaque instant - la totalité, mais comme événement spatio-temporel et de chair et de sang, moment vécu. L'eros platonicien est la recherche du «bien en soi », une notion que l'épistémologie nietzschéenne, par une analyse psychosociale suffisamment connue pour ne pas y revenir, abhorre. ${ }^{5}$ Dans l'acceptation de la réalité «telle qu'elle est », ce qui implique notamment, pour le philosophe, d'accepter ses propres limites, il me semble que Nietzsche désigne cette capacité chez un individu de ne jamais succomber, au nom de l'accroissement de la capacité d'expression de la vie (formule au fond spinozienne), à l'illusion sémantique. «Celui qui ne sait pas mettre sa volonté dans les choses veut au moins leur donner un sens : c'est ce qui le fait croire qu'il y a déjà une volonté en elles (Principe de la foi) » (Crépuscule, «Maximes et pointe $», \S 18)$.

Il ne s'agit donc pas chez Nietzsche d'un amour de la sagesse qui s'exprimerait dans la contemplation des Idées et d'une prudence par rapport à la valeur de cette activité, ou encore qui se réaliserait dans une orfèvrerie philosophique offrant ses bijoux à son propre contentement. La sagesse n'est jamais atteinte — elle est un projet, et c'est cela même, très précisément, qui est aimable. Il s'agit d'un amour du «sens de la sagesse » (Ecce Homo, «Préface », § 4), plutôt que d'un amour de la sagesse qui serait vénération des formes pures par-delà notre incapacité à y 
croire. Il s'agirait donc chez Nietzsche non pas « d'ouvrir un crédit» aux formes pures, mais de dissoudre la question même du crédit. ${ }^{6}$ « Je suis la bise des figues mûres », disait Zarathoustra. Le sens de la sagesse exige, me semble-t-il, de n'être pas fixé quant à l'usage du bien et du mal. C'est en cela que fatum est objet d'amour - car chez Nietzsche eros n'est pas fixé quant à l'usage du bien et du mal (il ne s'agit donc pas d'un amour de la pureté comme supposition).

Bien sûr, Daniel Tanguay aborde cette question. Il le fait sous le libellé de la «probité intellectuelle » (109). Il différencie cette dernière d'un «amour de la vérité » qui, contrairement à la probité intellectuelle athée, ne serait pas dogmatique. Je crois avoir démontré que Nietzsche, au moins dans ce qu'il en dit, n'est pas athée au sens dogmatique. L'amor fati de Nietzsche ne se distingue pas de l'amour grec de la vérité par son dogmatisme. Il se distingue plutôt, il me semble à tout le moins que cela est beaucoup plus caractéristique de l'originalité de la pensée de Nietzsche, en ce qu'il ne se contente pas d'en accepter par modestie l'opacité, mais décide par amour et pour des siècles et des siècles de se jeter à son cou. Cela s’indique déjà de soi, j’y reviendrai, mais il semble déjà qu'il n’y ait pas ici de hiérarchie, il n’y a que du désir — désir de laisser parler en soi l'inéluctable, faire confiance à l'expression, abandonner, suivre. Le courage du désir, c'est peut-être en fin de compte cela la noblesse nietzschéenne; une disposition particulière de la vie individuelle tardive face au temps (un devenu du devenir) et non un fait de nature qui se déploie de manière programmée dans le vivant (une nature philosophe, sa fausse modestie et ses objets éternels). «Ce n'est pas le doute, c'est la certitude qui rend fou » (Ecce Homo II, § 4).

Deuxièmement, il y a une certaine proximité entre amor fati et l'eros platonicien qui reste à dégager. On trouve à mon avis cette proximité sous le thème de la prudence. « Se jeter au cou » de l'opacité n'est peut-être pas en effet une excitation déraisonnable, révolutionnaire, mais, c'est 
ce que je suggère ici, une forme très fine de prudence philosophique (ou devrait-on peut-être dire de prudence vitale). Il s'agit, pour le philosophe qui aime le destin, non pas de se garder de se prononcer sur l'existence d'un Dieu biblique ou d'un ordre éternel tout en le supposant par amour, mais plutôt d'être «toujours à la hauteur du hasard. » Cet énoncé de la préface de Ecce Homo est particulièrement important, donnant à entendre que le chaos est chez Nietzsche enfin «accueilli » ou, si l'on veut, « révélé ». C'est une question de perspective : l'amour grec de la vérité succombe à l'idéal sans y croire (ce qui fait de la vie philosophique un mélange de prudence ésotérique et de mensonge exotérique), l'amour du destin croit sans succomber à la vérité (ce qui fait de la vie philosophique une expérience créatrice, dangereuse et certes tragique de la condition humaine). Dans les deux cas, il me semble trouver une ouverture mesurée à la totalité — et donc une prudence philosophique.

La mesure, dans le cas de Nietzsche, c'est de garder une certaine foi, la foi du dépassement créateur dont est capable l'animal humain. C'est une mesure d'hygiène devant prévenir la Création de nihilisme. La mesure, chez Platon/Strauss/Tanguay, c'est de ne pas succomber à une certaine foi, celle qui ferait des philosophes de véritables dieux sur terre. C'est une mesure d'hygiène devant prévenir l'ego pensant de totaliser la Création (Platon-maman et Hegel-meurtrier). Cette dernière option aboutit souvent à une sorte de modestie cynique qui préfère se dire ironique parce qu'elle a l'âme légère lorsqu'elle jouit au banquet, ou lorsqu'elle jouit de son ascétisme. Le type de la prudence de Nietzsche est difficile à percevoir, car différent du type de la prudence conservatrice — c'est une prudence structurale. Il y a partout le piège des mots sur la route vers l'homme (et peut-être pas tellement le piège de l'homme sur la route des mots, comme on aurait tendance à le croire). Amor fati, amour du destin. Sans reste. Nietzsche a 
un seul danger : le dégoût de l'homme. Ce qu'il a peut-être alors voulu dire c'est : croyez-moi, l'espoir est sauf — seulement, il est resté dans la boîte.

Maintenant, est-ce que cela nous laisse à penser que cette quête particulière de noblesse qu'est l'amor fati nietzschéen est dogmatique, posant, en croyant y échapper, un absolu? Est-ce que l'amor fati en tant que critère de la noblesse est affirmation d'une hiérarchie naturelle? Qui plus est, est-ce que cet amor fati souhaite «promouvoir l'inégalité naturelle »? Pour répondre tout de suite en partie à cette dernière question, je pense qu'il y a un problème dans la formulation straussienne du problème offerte par Tanguay. Nietzsche, à ma connaissance, n’utilise pas l'expression «inégalité naturelle». Quant à la promotion de l'inégalité, je me demande 1. comment on sentirait le besoin de promouvoir un fait naturel, puisqu'il est naturel, et 2. comment l'égalité serait-elle dans la pensée nietzschéenne moins naturelle que l'inégalité, si, comme le dit Nietzsche, la conscience n'est qu'une surface (Ecce Homo) et si, qui plus est, l'organisation humaine est affaire de valeurs, et que les valeurs sont créées ex nihilo dans les relations de pouvoir (Généalogie)? J'estime que Nietzsche n'a pas été assez bête, car il s'agit bien ici de bêtise, pour dire d'une part qu'il y a, fait métaphysique, des inégalités naturelles, et qu'il faut, prescription politique, les promouvoir. Les interprètes crapuleux de Nietzsche, conservateurs, fascistes italiens et national-socialistes, lui ont certainement fait défendre ce genre de positions. J'estime que c'est une simplification de la pensée de Nietzsche qui est à la fois inintéressante et indigne de la discussion philosophique - si toutefois on décide de prendre l'auteur au sérieux (ce qui n'est pas obligatoire).

Il me semble cependant que Nietzsche fait une double déclaration sur la question de l'inégalité, et il me faut en rendre compte. D’abord, et c'est le fait des œuvres de jeunesse, Nietzsche constate qu'il y a toujours eu, historiquement, des maîtres et des esclaves. La nature 
factuelle de cet argument (plutôt crédible) ne le qualifie pas en regard de la critique de StraussTanguay. Ensuite, Nietzsche affirme qu'il y a des volontés fortes (des philosophes, des saints, des artistes, des prêtres, des tyrans, des créateurs) et des volontés faibles (le troupeau, une matière). Ce serait alors sa manière de dire qu'il y a des «inégalités naturelles ». Il s'agit ici au mieux, me semble-t-il, si ce n'est d'un argument historique, d'un argument ontologique et non d'un argument métaphysique. Il reste à voir si celui-ci se qualifie pour la critique, ce qui me demande d'aborder directement la question de la relation entre nature et volonté de puissance, ou, pour le poser dans les termes plus spécifiques adoptés plus haut, entre hiérarchie naturelle et amor fati.

\section{2. Nature et volonté de puissance : Nietzsche a-t-il des idoles?}

Mon argument pour défendre Nietzsche de métaphysique sera de montrer que la philosophie nietzschéenne ne contient même pas, en fait, une ontologie (et ainsi encore moins une métaphysique), créant de toute pièce une pratique d'évitement éternellement recommencée de détermination de l'être présentée comme une décision, une décision courageuse parce qu'érotique, et ainsi noble. La philosophie de Nietzsche ne peut contenir une ontologie, et je devrai revenir sur le sens de la vie philosophique chez Nietzsche, parce que c'est une philosophie qui est de bout en bout expérimentale — et qui, au terme, ne s'adresse qu'à Nietzsche lui-même. ${ }^{7}$

«Je me raconte à moi-même ma vie »(Ecce Homo). La question d'une «nature », dans la formulation des problèmes qui occupent Nietzsche, est un non-lieu.

Je dois avant tout aborder de front une tension dérangeante posée au cœur de la critique de Nietzsche faite par Strauss-Tanguay. Nietzsche est du côté droit accusé d'historicisme (le monde est autocréation chaotique et il n'y a d'archè que voulue), et accusé de dogmatisme 
métaphysique du côté gauche (il y a une vision de la hiérarchie naturelle qui se réalise dans l'expression de la vie par les natures nobles). ${ }^{8}$ Ces deux éléments sont deux faces possibles (et communément invoquées) de la pensée de Nietzsche, et ces éléments relèvent de la question de la vérité. Ils relèvent également d'une vision particulière de la philosophie, celle où l'on demande : pourquoi y a-t-il quelque chose plutôt que rien? Cette question oblige de répondre qu'il y a quelque part ou un principe organisateur, ou un fait fondamental de l'être, puisqu'il y a effectivement quelque chose. On aura appelé métaphysiciens ceux qui ont prétendu pointer du doigt ce principe (un invisible généralement rendu lumineux à l'aide de mots sur du papier) et dialecticiens ceux qui prétendent atteindre ce fait fondamental. On appelle désormais historicistes ceux qui prétendent qu'il n'y a pas de principe organisateur qui soit primordial, et postmodernes ceux qui récusent le fait même d'une identité de l'être, le monde réalisé se donnant lui-même comme principe organisateur, l'être n'étant que le résultat d'une mise en discours qui est immanente au champ des forces sociales. Ce sont ces constats à partir desquels on conclura qu'il n'est pas possible de poser une hiérarchie des principes, non plus que de dire l'Être.

La vérité. Je veux suggérer que la vision nietzschéenne de la philosophie se pose génétiquement après ces constats. Cette vision ne pose pas la question du principe premier et de l'Être (pourquoi y a-t-il quelque chose plutôt que rien?), mais demande plutôt : pourquoi y a-t-il ceci plutôt que cela? C'est ici la question du fleurissement, du jeu des oppositions créatrices (elle relèverait de l'éthique, de la morale, et de la politique, mais aussi de la «psychologie », de la philosophie de la culture, du fantasme de vie) et non la question du néant (qui relève de la logique, de la métaphysique et de l'ontologie, mais aussi du fantasme littéraire, du fantasme de mort). Le fait qu'il y ait un monde plutôt que rien ne fait pas partie des objets de la spéculation philosophique, chez Nietzsche. Le critère de la noblesse, amor fati, indique que l'on mesure la 
valeur d'un homme à la quantité de vérité qu'il peut supporter. Nietzsche le philosophe, avec une sorte de réalisme florentin appliqué à l'âme humaine, récuse l'idée du Néant. C'est une fausse question, une mascarade de la peur de mourir du philosophe, une «objection contre la vie ». Nietzsche peut amorcer la philosophie en la fondant sur le fait de l'expérience charnelle de sa vie. Il y a quelque chose, cela est digne d'amour, et notre tâche est d'approfondir notre capacité d'affecter et d'être affecté par ce quelque chose.

La critique de l'objectivité faite par Nietzsche (notamment dans les premiers morceaux du Crépuscule) suggère en ce sens que la soumission à un seul principe organisateur est un appauvrissement de la capacité de percevoir ce qui est. À l'objectivité, Nietzsche préfère le perspectivisme (Généalogie III, § 12). Il ne s'agit pas seulement d'«historicisme », et pas du tout de «relativisme »: il n'y a pas d'abdication de la conquête du réel dans l'épistémologie nietzschéenne (que l'on retourne à son hommage à Bacon dans Ecce Homo). Il s'agit plutôt de faire l'expérience du monde en mettant en jeu le plus de principes organisateurs possible, autant qu'il est possible à un homme d'en supporter. Le philosophe doit se faire le siège, l'expérimentateur des nervures du monde — ce serait la seule manière de saisir un monde — et agir sur ce monde dont la forme est la volonté accaparant la matière, s'accaparant ... et ce temps qui passe. Il n’y a pas de déprise épistémologique relativiste dans le perspectivisme nietzschéen. Il s'agit plutôt d'une technique cognitive qui assimile et synthétise les constats de l'historicisme. ${ }^{9}$

La volonté. Cela nous renvoie ainsi à l'autre face de la critique de Strauss-Tanguay, expliquant que Nietzsche aurait commis une métaphysique en proposant de comprendre le monde à l'aide de la «volonté de puissance ». Établissons d'emblée que Nietzsche admet effectivement l'existence de principes organisateurs — et il admet non pas tant leur relativité que 1. leur coexistence, et 2 . leur qualité d'artefact. Il faut ici percevoir le niveau d'intégration de 
l'idée de principes organisateurs dans la pensée nietzschéenne. Ils sont admis en tant que faits d'observation, et même en tant que faits matériels. La qualité d'artefact des principes organisateurs indique que ce sont toujours des effets, qu'ils sont le fait de l'homme (et de l'individu Nietzsche s'il y a lieu). Leur coexistence implique qu'aucun n'est nécessaire en regard d'une quelconque nature et que s'il y a un bien un monde réel, celui-ci n'est qu'en tant qu'il est résorption des mondes possibles, en tant qu'il se présente comme pérenne (rapt du futur éternellement recommencé).

La réalité symbolique du monde (architecture cognitive et morale), c'est tout l'effort de Nietzsche dans la Généalogie de démontrer cette chose, est un fait relationnel historique : c'est l'inscription contingente infiniment recommencée des rapports de force en tant que cette inscription se manifeste dans la création perpétuée des consciences. Conceptuellement, Nietzsche explique que la conscience est une « surface » (Ecce Homo), voulant dire par là que l'inscription y est tout, voulant dire par là que rien ne préexiste à cette conscience qui puisse être dit — et que tout ce qui s'y inscrit est le fait de la chair, de l'histoire, et plus encore le fait relationnel par excellence. La conscience est la trace des combats proliférant sur toutes les surfaces de ce qui est et étant de tout ce qui arrive, principe polaire admis non pas comme «naturel », mais admis comme effet général du fait qu'il y a quelque chose plutôt que rien. Notre vie est, comme le dit en quelque sorte Witold Gombrowicz, l'histoire de notre déformation.

Disant cela, admettant comme effet de la vie un certain principe polaire, Nietzsche, si on le lit en tant que sa vie a été un travail de formulation d'une pensée en mouvement, ne pose pas une archè du monde qui doive être reconnue comme finale et célébrée. Il s'agit pour Nietzsche, à propos de ce principe polaire, de faits de son expérience du monde, de la description minutieuse de la texture de ce qu'il a expérimenté comme sa propre volonté, disputée sans cesse entre sa 
propre vie et sa propre mort, ses propres défaites, ses propres victoires. Celui qui pense ose penser l'universel à travers sa propre expérience du monde (Gai savoir I, § 2) — et l'expérience de Nietzsche est celle de cette polarité proliférante et indécrottable, celle de l'organisation de constellations mouvantes à l'intérieur d'une seule vie, d'un seul corps, nœud où s'agglutine un ensemble foncièrement instable et infiniment reconstitué en états variables. «La multiplicité des états intérieurs est chez moi extraordinaire » (Ecce Homo II, § 4). Et dans Humain trop humain I $(\S 14):$

Toutes les vibrations assez intenses de l'âme provoquent une résonance d'impressions et d'états analogues; elles fouillent pour ainsi dire la mémoire. Elles suscitent en nous quelque réminiscence, éveillent la conscience d'états semblables et de leur origine. Il se forme ainsi de promptes et familières associations de sentiments et d'idées, que l'on finit, lorsqu'elles se succèdent à la vitesse de l'éclair, par ne plus même percevoir comme des complexes, mais bien comme des unités. C'est en ce sens que l'on parle de sentiment moral, de sentiment religieux, comme s'il s'agissait d'authentiques unités : ce sont en vérité des fleuves avec des centaines de sources et d'affluents. Là non plus, comme c'est souvent le cas, l'unité du mot ne garantit en rien l'unité de la chose.

La réponse à la question «Pourquoi y a-t-il ceci plutôt que cela? » est ainsi subsumée dans l'idée de la volonté de puissance, qui est la désignation scripturale d'une réalité existentielle singulière, mystérieuse, celle de l'expérience de vie d'un individu, Friedrich Nietzsche. Il n'y a pas de secret transcendantal, la singularité caractérielle de la philosophie de Nietzsche est avouée, et même tournée en dérision. Être satyre plutôt que saint ... voici l'homme, a-t-on dit en s'en lavant les mains. La volonté de puissance est un principe toujours posthume, si l'on peut dire, chez Nietzsche, qui se désole de voir que l'on brisera plus facilement la jambe que le mot.

L'éthique. C'est l'expérience de pensée qui est le propre de la vie philosophique nietzschéenne, celle de ceux qui non pas osent savoir, mais osent créer. Les déclarations à l'emporte-pièce sur la transformation du monde par les philosophes du futur, que l'on trouve 
concentrées dans Par-delà bien et mal, sont peut-être (lecture possible parmi les lectures) une expérience de pensée réalisée par Nietzsche. Cette expérience serait alors celle du télescopage des états intérieurs à la grandeur d'une civilisation, elle est aussi le revers de la bonté zarathoustréenne : «... le livre n’a pas un seul mot de bienveillance ... c'est Dieu lui-même qui, sous l'apparence du serpent, s'est reposé à la fin de sa tâche sous l'arbre de la connaissance » (Ecce Homo III, «Par-delà bien et mal», §2). Dans Par-delà bien et mal, Nietzsche est l'Europe, quelque chose comme un croisement entre Napoléon et Héraclite. Il s'agit d'un jeu — car ce que l'on fait, amor fati, on doit le faire en jouant (Beispiel). Il s'agit d'un modèle de la tentation — d'une expérience fantasmatique et thérapeutique du politique.

Chez Nietzsche, s'il y a un naturalisme, c'est dans son immoralisme qu'il s'exprime (le mal est aussi naturel que le bien chez l'homme). S'il y a un naturalisme, donc, c'est celui du principe polaire en tant que Nietzsche l'expérimente comme individu (destin). Ce principe n'est en revanche pas «premier», ne révélant pas une hiérarchie naturelle — dans la dialectique morale historique le bien étant un ancien mal et le mal un futur bien (Gai savoir I, § 2). La hiérarchie est à l'intérieur de chacun de nous — c'est une organisation aléatoire qui répond à la vie comme phénomène spontané, autogénéré, constituant et constitutif. Ainsi parlait Zarathoustra est bonté, Par-delà bien et mal est malveillance. Le Diable prend le relais d'un Dieu fatigué — Nietzsche reprend dans l'écriture les motifs des divinités grecques.

La noblesse est le courage de soi, le courage de son immoralisme, le courage de ne pas projeter ses peurs dans le monde sous la forme de la négation de la vie — plutôt son affirmation brutale, thérapeutique. Il n'y a pas tellement hiérarchie que polarisation entre forces et faiblesses, entre vie et mort, et ce, non pas seulement dans le monde, mais surtout dans l'âme, et a fortiori, chez Nietzsche, dans l'écriture. C'est l'expérience du principe polaire qui est première et 
éternellement recommençable — et elle est le mode du connaître libre de la mort en tant que c'est ainsi que nous en faisons l'expérience à travers la vie de l'homme Nietzsche, hic et nunc. La noblesse n'est pas une recommandation de la nature, elle est espoir. La noblesse n'est pas une recommandation de la nature, elle est une recommandation de Nietzsche, livrant un combat, aimant dans l'homme le geste même du sacrifice.

Soit votre amour de la vie un amour de votre espérance la plus haute, et que votre espérance la plus haute soit de la vie la plus haute pensée!

Mais votre plus haute pensée, vous devez de moi-même en recevoir le commandement - et la voici : l'homme est quelque chose qui doit être surmonté.

Votre vie d'obéissance et de guerre, de la sorte la vivez! Qu'importe une longue vie? Est-il guerrier qui veuille être épargné?

Point ne vous épargne, foncièrement je vous aime, ô mes frères dans la guerre! (Zarathoustra, « De la guerre et des guerriers »)

Il n’y a en fait même pas de Tout révélé ou supposé chez Nietzsche, il n’y a que du devenir, et la tâche est de l'accueillir infiniment. Nietzsche est un temporel, il n'est pas un spatial. Son rapport à l'espace n'est pas le cadastre, la mesure, le nomos, la métaphysique, la loi (la désignation d'une limite, le rappel d'une mort imminente). L'espace chez Nietzsche c'est la vénération primitive (un seul soleil, une seule terre) et la distanciation («du haut des cimes »). L'espace est le prétexte de la philosophie, le prétexte de l'union de la vie et du temps. «... Je ne réfute pas les idéaux, je me contente de mettre des gants ... »(Ecce Homo, «Préface », § 3). Il y a un passage à l'éthique dans l'œuvre de maturité, et enfin un passage à l'hygiène. Entre historicisme et métaphysique, Nietzsche se ménage une voie de sortie : il s'agit de ne pas prendre sa propre parole au sérieux (Heiterkeit), de ne pas emmurer, impératif éthique, le futur dans le présent. La vérité n'est pas récusée, elle est toujours remise à plus tard. Elle est un don au futur sans cesse recommencé. 
À ce point, si on refuse toujours à Nietzsche la validité de son exploration hors de la métaphysique sous prétexte qu'il dit quelque chose (il prétend à une vérité), — on disqualifie sa propre critique, car on est alors en train de dire qu'à chaque fois que l'on parle, on commet de la métaphysique. C'est bien possible, et je pense que Nietzsche l'a compris mieux que personne jusqu'à présent, mais alors tout cela ne veut plus rien dire. Il y a une invitation chez Nietzsche et, en fin de compte, il s'agit avant tout de se laisser séduire, de « suivre », et d'être capable à la fois de «savoir se garder». Courage et prudence dans l'exploration de la forme du temps. Nietzsche savait que cette tâche était celle des rares.

\section{Conclusion : le topos meurtrier ou « si j'te pogne, j’te mange »}

L'individu souverain ne fait pas porter l'évaluation sur les équivalences entre les biens (marché) ou entre dommage et souffrance (justice) comme les nobles barbares, il ne se donne pas de créditeurs abstraits qui prendraient en charge le formatage de la matière humaine (les formes pures, ou Dieu) comme les philosophes socratiques ou les prêtres. Il se promet à lui-même la canalisation noble de ses désirs dans un surplus créateur qui ne lui appartient pas — don absolu, fission du rapport de force originel. La rencontre entre Nietzsche et Strauss lui-même semble avoir constitué une rencontre importante et difficile pour ce dernier. Pour cause : la pensée de Nietzsche non seulement foule aux pieds la conception platonicienne de la vie philosophique, mais semble avoir les moyens d'esquisser une alternative. Elle indique que le noble mensonge est une supercherie - et que l'idée même du Bien de la communauté n'est que le dispositif couard de reproduction de la caste philosophique et religieuse - le fait d'individus sadomasochistes, en tous cas d'individus enfermés dans les catégories qui sont celles de l'échange, et qui sont anthropologiquement antérieures à toute formulation de la liberté d'une vie 
individuelle — qui s'indique d'elle-même dans le don créateur, qui internalise la violence de la civilisation et la détourne au profit de l'espoir. Au profit d'un devenir.

Daniel Tanguay réagit dans son texte au fait que, dans les dernières décennies, un débat interne à l'école straussienne tend à imputer à Strauss des affinités fondamentales avec la pensée nietzschéenne. De point de vue de l'histoire des idées, la question importante ici ne serait pas, au fond, de savoir si Strauss est nietzschéen ou non. Ce qui s'impose à l'observation est que c'est le mouvement même de la pensée politique au $\mathrm{XX}^{\mathrm{e}}$ siècle qui est inéluctablement confronté au diagnostic nietzschéen, et qu'une fois que ce diagnostic contamine le philosophe platonicien, celui-ci est pris au piège. La pensée se trouve irrémédiablement sur un fil de tension : nomos/Chronos, Être/faire, fixité/mouvement, Eros/amor.

Chez Nietzsche, le déplacement de la question philosophique, sa mise en mouvement, sa qualité de langage comme vibration du monde, constitue un topos meurtrier. Je concède que je gravite dans la constellation discursive nietzschéenne, et que cette conversation avec StraussTanguay n'a pas vraiment ici lieu. Cependant, à moins justement de s'enfermer dans le vocabulaire strausso-platonicien, ce qui est une option possible, la rencontre avec le texte nietzschéen oblige à considérer (ce qui ne signifie pas y prendre part) un destin autre de la vie philosophique. Pourquoi ceci plutôt que cela? Nous sommes devant un dilemme terrible, qui est celui de trahir la civilisation occidentale — de tenter l'aventure de mondes possibles. On ne peut plus l'éviter, et nous en sommes la preuve, Strauss, ses interprètes, Daniel Tanguay et moi. Qu'est-ce que cela signifie? Nietzsche dit : «Dire la vérité et bien décocher ses traits, c'est la vertu persane. - Me comprend-on?... » (Ecce Homo IV, § 3). 


\section{Notes}

1 Sur cette question du don et de la parole chez Nietzsche, voir Giroux, «Nietzsche et Sloterdijk ».

2 Paolo Valesio souligne dans une ébauche de phénoménologie érotique de la vie politique pardelà bien et mal le fait que Zarathoustra est au fond un masturbateur — si le fascisme, par principe polaire, est viol.

${ }^{3}$ Passons sur le fait que la prudence pourrait être comprise comme le dogme de l'indécision. C'est un argument formel que Strauss utilise d'ailleurs contre le relativisme. Passons également sur le fait que la volonté de puissance est peut-être aussi, chez Nietzsche, supposée plutôt qu'affirmée (en vertu du fait que la volonté de puissance est toujours traitée chez Nietzsche comme un effet, effet que Nietzsche désigne comme étant la «Vie», idée qui recoupe un avènement difficile à réfuter hors du nihilisme métaphysique que je juge inutile de défaire).

${ }^{4}$ L'avant-propos de Ecce Homo me semble être un extrait particulièrement pertinent à notre problématique.

${ }^{5}$ La troisième dissertation de la Généalogie de la morale, le Crépuscule des idoles et les 39 premiers aphorismes de L'Antéchrist constituent le cœur de cette analyse psychosociale. Évidemment, entre la critique de l'idéalisme et la réalisation de son contraire, le travail de la pensée de Nietzsche est plus brumeux. Il demeure que cette pensée fait signe vers une telle réalisation.

${ }^{6}$ Sur la relation originelle créditeur/débiteur et le transfert du crédit universel au Dieu unique, voir Généalogie II, particulièrement $§ 1-12$.

${ }^{7}$ Les interprétations de l'œuvre nietzschéenne qui développent une ontologie de Nietzsche, et au premier chef l'interprétation de Heidegger, sont généralement fondées sur les écrits non publiés de Nietzsche, et en particulier sur les fragments réunis sous le titre Volonté de puissance. La décision d'intégrer ou d'exclure les écrits non publiés, tel que cela a été démontré par Linda Williams, a en effet une influence décisive sur l'interprétation du statut du travail de Nietzsche. Ma propre interprétation se situe résolument du côté de l'usage quasi-exclusif des écrits publiés, laissant de côté la notion de volonté de puissance comme moment synthétique de la pensée de Nietzsche.

8 Je trouve que le fait de jouer sur ces deux tableaux dans une seule critique indique une illocution perverse: il s'agit de défaire Nietzsche à tout prix. On accorde ce faisant beaucoup de valeur à la pensée de Nietzsche, on accorde aussi beaucoup de valeur à sa propre situation d'énonciation. 
${ }^{9}$ La guerre des mots est quelque chose de sérieux pour Nietzsche - il n'y a pas de béatitude relativiste en aucun point de son travail, publié ou non. Je renvoie à mon interprétation du passage célèbre et aphorisme carrefour de «Vérité et mensonge extra-moral » sur les langages comme des armées de métaphores (Giroux, «L'unité »). Les tenants de l'épistémologie relativiste trouvent dans ce passage l'argument final de leur position. J'y vois une lecture du langage comme expérience — expérience dont la réalité (et la vérité) ne fait pas de doute pour Nietzsche.

\section{Ouvrages cités}

Conway, Daniel W., «Love's Labor's Lost: The Philosopher's Versucherkunst», in Salim Kemal (dir.), Nietzsche, Philosophy and the Arts, New York, Cambridge University Press, 1998, p. 287-309.

Giroux, Dalie, «L'unité de la forme et du fond et la grande politique nietzschéenne », Sociétés, vol. 81, 2003, p. 45-59.

—, « Nietzsche et Sloterdijk, corps en résonance », Horizons philosophiques, vol. 17, n², 2007, à paraître.

Nietzsche, F. W., La généalogie de la morale, trad. Isabelle Hildenbrand et Jean Gratien, Paris, Gallimard, 1971.

—, Ecce Homo, suivi de Nietzsche contre Wagner, trad. Éric Blondel, Paris, Flammarion, 1992.

—, Le gai savoir, trad. Alexandre Vialatte, Paris, Gallimard, 1950.

—, Humain trop humain, vol. 1, trad. Robert Rovini, Paris, Gallimard, 1987.

—, Humain trop humain, vol. 2, trad. Robert Rovini, Paris, Gallimard, 1988.

—, Ainsi parlait Zarathoustra, trad. Maurice de Gandillac, Paris, Gallimard, 1971.

—, Par-delà bien et mal, trad. André Meyer et René Guast, Paris, Hachette, 1948.

—, Le crépuscule des idoles, suivi de Le cas Wagner, trad. Henri Albert, Paris, Flammarion, 1985. 
Tanguay, Daniel, « Strauss disciple de Nietzsche? À propos d'une hypothèse récente sur le sens “caché” de l'œuvre de Leo Strauss », Les études philosophiques, vol. 1, 2000, p. 117-146.

Valesio, Paulo, «The Beautiful Lie : Heroic Individuality and Fascism », in Sosna Wellberg Heller (dir.), Reconstructing Individualism. Autonomy, Individuality, and the Self in Western Thought, Stanford (CA), Stanford University Press, 1986, p. 163-183.

Williams, Linda L., «Will to Power in Nietzsche's Published Works and the Nachlass », Journal of the History of Ideas, vol. 57, 1986, p. 447-463. 